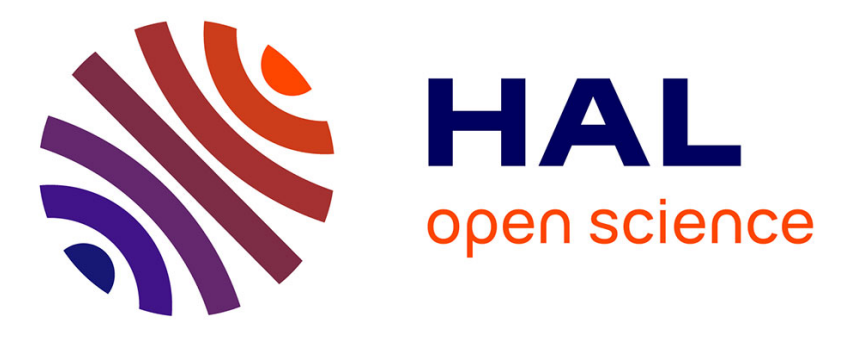

\title{
The impedance response of rotating disk electrodes
}

\author{
Shao-Ling Wu, Mark E. Orazem, Bernard Tribollet, Vincent Vivier
}

\section{To cite this version:}

Shao-Ling Wu, Mark E. Orazem, Bernard Tribollet, Vincent Vivier. The impedance response of rotating disk electrodes. Journal of electroanalytical chemistry and interfacial electrochemistry, 2015, 737, pp.11-22. 10.1016/j.jelechem.2014.05.036 . hal-01111367

\section{HAL Id: hal-01111367 \\ https://hal.science/hal-01111367}

Submitted on 30 Jan 2015

HAL is a multi-disciplinary open access archive for the deposit and dissemination of scientific research documents, whether they are published or not. The documents may come from teaching and research institutions in France or abroad, or from public or private research centers.
L'archive ouverte pluridisciplinaire HAL, est destinée au dépôt et à la diffusion de documents scientifiques de niveau recherche, publiés ou non, émanant des établissements d'enseignement et de recherche français ou étrangers, des laboratoires publics ou privés. 


\title{
The Impedance Response of Rotating Disk Electrodes
}

\author{
Shao-Ling $\mathrm{Wu}^{\mathrm{a}}$, Mark E. Orazem ${ }^{\mathrm{a}, *}$, Bernard Tribollet ${ }^{\mathrm{b}}$, Vincent Vivier ${ }^{\mathrm{b}}$ \\ ${ }^{a}$ Department of Chemical Engineering, University of Florida, Gainesville, FL, 32611, USA \\ ${ }^{b}$ LISE, UPR 15 du CNRS, Université P. et M. Curie, CP 133, 4 Place Jussieu, 75252 Paris cedex 05 France
}

\begin{abstract}
Classifications and recent findings are reviewed for numerical simulations describing the impedance response of rotating disk electrodes. The disk geometry is shown to cause a complex character to the ohmic contribution to the impedance response. For reactions that do not involve formation of adsorbed intermediates, the frequency dispersion associated with the disk geometry occurs above a characteristic frequency. Such dispersion may be avoided by selecting a disk of sufficiently small dimension. For reactions that do involve formation of adsorbed intermediates, a low-frequency dispersion is also seen. Models including the influence of mass transfer show that the coupling of faradaic and charging currents induces frequency dispersion at frequencies associated with the faradaic reaction.
\end{abstract}

Keywords: coupled faradaic and charging currents, frequency dispersion, CPE

\section{Introduction}

The disk electrode, consisting of a circular electrode embedded in an insulating plane, is commonly used in electrochemical measurements. The disk geometry may be associated with rotating or stationary electrodes. Newman showed, in 1966, that electrode-insulator interface gives rise to nonuniform current and potentials distributions for currents below the mass-transfer limited current. $[1,2]$ The geometry-induced nonuniform current and potential distributions influences the transient response of the disk electrode. Nisancioglu and Newman[3, 4] developed a solution for the transient response of a faradaic reaction on a non-polarizable disk electrode to step changes in current. The solution to Laplace's equation was performed using a transformation to rotational elliptic coordinates and a series expansion in terms of Lengendre polynomials. Antohi and Scherson have recently expanded the solution to the transient problem by expanding the number of terms used in the series expansion.[5]

Newman also provided a treatment for the impedance response of a disk electrode which showed that the capacity and ohmic resistance were functions of frequency above a critical value of frequency.[6] More recently, the present authors embarked on a comprehensive numerical and experimental study of the impedance response of the disk electrode, including the response of ideally polarized electrodes,[7] electrodes showing local CPE behavior,[8] electrodes with faradaic

\footnotetext{
* Corresponding author

Email address: meo@che.ufl.edu (Mark E. Orazem)

published as S.-L. Wu, M. E. Orazem, B. Tribollet, and V. Vivier, "The Impedance Response of Rotating Disk Electrodes," Journal of Electroanalytical Chemistry, 737 (2015), 11-22.
} 
reactions, [9] electrodes with reactions involving adsorbed intermediates,[10, 11] and electrodes subject to reactions influenced by mass transfer.[12] The authors also explored the origin of the complex ohmic impedance $[13,14]$ and provided experimental verification of the phenomena predicted by the simulations. $[15,16]$ The objective of this work is to provide an overview of the impedance of a disk electrode.

\section{Mathematical Development}

Deterministic impedance models for a disk electrode may be divided into two categories. The models presented in Section 2.1 consider that mass transfer is unimportant. The potential and current is either controlled by the ohmic resistance of the electrolyte, i.e., a primary distribution, or a combination of ohmic resistance and kinetic effects at the electrode, a secondary distribution. The models presented in Section 2.2 pertain to systems where mass transfer plays a significant role.

\subsection{Influence of Ohmic and Kinetic Phenomena}

Under the assumption that mass-transfer may be neglected, the electrical potential in the electrolyte is governed by Laplace's equation

$$
\nabla^{2} \Phi=0
$$

The system may be assumed to have cylindrical symmetry such that the potential in solution is dependent only on the radial position $r$ along the electrode surface and the normal position $y$. In response to an alternating current with a particular angular frequency $\omega=2 \pi f$, the potential can be separated into steady and time-dependent parts as

$$
\Phi=\bar{\Phi}+\operatorname{Re}\{\widetilde{\Phi} \exp (\mathrm{j} \omega t)\}
$$

where $\bar{\Phi}$ is the steady-state solution for potential and $\widetilde{\Phi}$ is the complex oscillating component which is a function of position only. Similarly, the applied potential can be expressed as

$$
V=\bar{V}+\operatorname{Re}\{\widetilde{V} \exp (\mathrm{j} \omega t)\}
$$

Therefore, the form of Laplace's equation to be solved, subject to appropriate boundary conditions, is

$$
\frac{1}{r} \frac{\partial}{\partial r}\left(r \frac{\partial \widetilde{\Phi}}{\partial r}\right)+\frac{\partial^{2} \widetilde{\Phi}}{\partial y^{2}}=0
$$

Equation (4) can be solved using numerical methods.

The boundary condition for potential at $r \rightarrow \infty$ is that $\widetilde{\Phi} \rightarrow 0$, and the boundary condition on the insulating surface is that $\partial \widetilde{\Phi} / \partial y=0$. The distinction between different conditions simulated is seen in the boundary condition for the electrode surface.

\subsubsection{Blocking Electrode}

For a blocking electrode, [7]

$$
\mathrm{j} K\left(\widetilde{V}-\widetilde{\Phi}_{0}\right)=-\left.r_{0} \frac{\partial \widetilde{\Phi}}{\partial y}\right|_{y=0}
$$


where $\Phi_{0}$ is the potential in the solution adjacent to the electrode and $K$ is the dimensionless frequency

$$
K=\frac{\omega C_{0} r_{0}}{\kappa}
$$

where $C_{0}$ is the double layer capacitance and $\kappa$ is the electrolyte conductivity. The current is only that required to charge or discharge the electrode.

\subsubsection{Blocking Electrode with CPE Behavior}

For a blocking electrode with CPE behavior,[8] the interfacial impedance is given as

$$
Z_{\mathrm{CPE}}=\frac{\widetilde{V}-\widetilde{\Phi}}{\widetilde{i}}=\frac{1}{(\mathrm{j} \omega)^{\alpha}} Q
$$

On application of the Euler identity,

$$
\mathrm{j}=\cos \left(\frac{\pi}{2}\right)+\mathrm{j} \sin \left(\frac{\pi}{2}\right)
$$

Thus

$$
K\left(\widetilde{V}-\widetilde{\Phi}_{0}\right)\left(\cos \left(\frac{\alpha \pi}{2}\right)+\mathrm{j} \sin \left(\frac{\alpha \pi}{2}\right)\right)=-\left.r_{0} \frac{\partial \widetilde{\Phi}}{\partial y}\right|_{y=0}
$$

where $\mathrm{K}$ is the dimensionless frequency defined for a system with $\mathrm{CPE}$ behavior to be

$$
\mathrm{K}=\frac{Q \omega^{\alpha} r_{0}}{\kappa}
$$

The definition of the dimensionless frequency presented in equation (10) differs from the definition expressed in equation (6).

\subsubsection{Electrode with Faradaic Reactions}

For an electrode with faradaic reaction, $[9]$ the boundary condition at the electrode is expressed in frequency domain as

$$
\mathrm{j} K\left(\widetilde{V}-\widetilde{\Phi}_{0}\right)+J\left(\widetilde{V}-\widetilde{\Phi}_{0}\right)=-\left.r_{0} \frac{\partial \widetilde{\Phi}}{\partial y}\right|_{y=0}
$$

where $\widetilde{V}$ represents the imposed perturbation in electrode potential referenced to an electrode at infinity and $K$ is defined by equation (6) under the assumption of pure capacitive behavior for the double layer. Under the assumption of linear kinetics, valid for $\bar{\imath}<<i_{0}$, the parameter $J$ is defined to be

$$
J=\frac{\left(\alpha_{\mathrm{a}}+\alpha_{\mathrm{c}}\right) \mathrm{F} i_{0} r_{0}}{\mathrm{R} T \kappa}
$$

For Tafel kinetics, valid for $\bar{\imath}>>i_{0}$, the parameter $J$ is defined to be

$$
J\left(\bar{V}-\bar{\Phi}_{0}\right)=\frac{\alpha_{\mathrm{c}} \mathrm{F}\left|\bar{i}\left(\bar{V}-\bar{\Phi}_{0}\right)\right| r_{0}}{\mathrm{RT} \kappa}
$$

As $\bar{\Phi}_{0}$ depends on radial position, the value of $J$ expressed in equation (13) is a function of radial position. 
The charge-transfer resistance for linear kinetics can be expressed in terms of parameters used in equation (12) as

$$
R_{\mathrm{t}}=\frac{\mathrm{R} T}{i_{0} \mathrm{~F}\left(\alpha_{\mathrm{a}}+\alpha_{\mathrm{c}}\right)}
$$

and, in terms of parameters used in equation (13),

$$
R_{\mathrm{t}}=\frac{\mathrm{RT}}{\left|\bar{i}\left(\bar{V}-\bar{\Phi}_{0}\right)\right| \alpha_{\mathrm{c}} \mathrm{F}}
$$

For linear kinetics, $R_{t}$ is independent of radial position, but, under Tafel kinetics, as shown in equation (15), $R_{t}$ depends on radial position. From a mathematical perspective, the principal difference between the linear and Tafel cases is that $J$ and $R_{\mathrm{t}}$ are held constant for the linear polarization; whereas, for the Tafel kinetics, $J$ and $R_{\mathrm{t}}$ are functions of radial position determined by solution of the nonlinear steady-state problem.

Huang et al. [9] provide a relationship between the parameter $J$ and the charge-transfer and ohmic resistances as

$$
J=\frac{4}{\pi} \frac{R_{\mathrm{e}}}{R_{\mathrm{t}}}
$$

Large values of $J$ are seen when the ohmic resistance is much larger than the charge-transfer resistance, and small values of $J$ are seen when the charge-transfer resistance dominates. The definition of parameter $J$ in equation (16) is the reciprocal of the Wagner number[17], which is a dimensionless quantity that measures the uniformity of the current distribution in an electrolytic cell.

\subsubsection{Electrode with Faradaic Reactions Coupled by Adsorbed Intermediate}

As the reaction sequences become more complicated, it becomes difficult to express the model results in a general manner. Wu et al. [10] analyzed the impedance response associated with two successive charge-transfer steps involving an intermediate species adsorbed on the electrode surface, i.e.,

$$
\mathrm{M} \rightarrow \mathrm{X}_{\mathrm{ads}}{ }^{+}+e^{-}
$$

and

$$
\mathrm{X}_{\mathrm{ads}}^{+} \rightarrow \mathrm{P}^{2+}+e^{-}
$$

The reactant could be a metal $\mathrm{M}$ which dissolves to form an adsorbed intermediate $\mathrm{X}_{\mathrm{ads}}{ }^{+}$, which then reacts to form the final product $\mathrm{P}^{2+}$. The reactions were assumed to be irreversible, and diffusion processes were considered negligible. Similar mechanisms were proposed by Epelboin and Keddam[18] for calculating the impedance of iron dissolution through two steps involving an adsorbed $\mathrm{FeOH}$ intermediate, and by Peter et al. [19] for the impedance model of the dissolution of aluminum in three consecutive steps with two adsorbed intermediates.

Under the assumption of Tafel kinetics, the steady-state current densities for reactions (17) and (18) may be expressed by

$$
\bar{i}_{\mathrm{M}}=K_{\mathrm{M}}(1-\bar{\gamma}) \exp \left[b_{\mathrm{M}}\left(\bar{V}-\bar{\Phi}_{0}\right)\right]
$$

and

$$
\bar{i}_{\mathrm{X}}=K_{\mathrm{X}} \bar{\gamma} \exp \left[b_{\mathrm{X}}\left(\bar{V}-\bar{\Phi}_{0}\right)\right]
$$

where $K_{\mathrm{M}}$ and $K_{\mathrm{X}}$ are effective rate constants, with units of current density, for reactions (17) and (18), $\gamma$ is the fractional surface coverage by intermediate $\mathrm{X}_{\mathrm{ads}}{ }^{+}$, and $b_{\mathrm{M}}$ and $b_{\mathrm{X}}$ are $\alpha_{\mathrm{M}} F / R T$ and 
$\alpha_{\mathrm{X}} F / R T$ where $\alpha_{\mathrm{M}}$ and $\alpha_{\mathrm{X}}$ are transfer coefficients for reactions (17) and (18), respectively. The variation of surface coverage is given by

$$
\dot{\gamma}=\frac{d \gamma}{d t}=\frac{i_{\mathrm{M}}-i_{\mathrm{X}}}{\Gamma F}
$$

where $\Gamma$ is the maximum surface concentration of $X_{\text {ads }}^{+}$. The steady-state condition is defined by $d \gamma / d t=0$; thus, the expression of steady-state surface coverage can be calculated from equations (19) and (20) to be

$$
\bar{\gamma}=\frac{K_{\mathrm{M}} \exp \left[b_{\mathrm{M}}\left(\bar{V}-\bar{\Phi}_{0}\right)\right]}{K_{\mathrm{M}} \exp \left[b_{\mathrm{M}}\left(\bar{V}-\bar{\Phi}_{0}\right)\right]+K_{\mathrm{X}} \exp \left[b_{\mathrm{X}}\left(\bar{V}-\bar{\Phi}_{0}\right)\right]}
$$

The steady-state surface coverage is useful for interpretation of the simulated impedance response.

The current at the electrode surface can be written by use of the reaction kinetics developed in equations (19) and (20) and expressed in frequency domain as

$$
K j\left(\widetilde{V}-\widetilde{\Phi}_{0}\right)+J_{1}\left(\widetilde{V}-\widetilde{\Phi}_{0}\right)+J_{2}\left(\widetilde{V}-\widetilde{\Phi}_{0}\right)=-\left.r_{0} \frac{\partial \widetilde{\Phi}}{\partial y}\right|_{y=0}
$$

where $\widetilde{V}$ represents the imposed perturbation in electrode potential referenced to an electrode at infinity and $K$ is the dimensionless frequency defined by equation (6). The parameters $J_{1}$ and $J_{2}$ are dimensionless functions dependent on angular frequency and radial position on the electrode surface, i.e.,

$$
J_{1}(r, \omega)=J_{\mathrm{M}}(r)-\frac{\frac{\left|\bar{i}_{\mathrm{M}}(r)\right|}{1-\bar{\gamma}(r)}\left[J_{\mathrm{M}}(r)-J_{\mathrm{X}}(r)\right]}{\Gamma F j \omega+\frac{\left|\bar{i}_{\mathrm{M}}(r)\right|}{1-\bar{\gamma}(r)}+\frac{\left|\bar{i}_{\mathrm{X}}(r)\right|}{\bar{\gamma}(r)}}
$$

and

$$
J_{2}(r, \omega)=J_{\mathrm{X}}(r)-\frac{\frac{\left|\bar{i}_{\mathrm{X}}(r)\right|}{\bar{\gamma}(r)}\left[J_{\mathrm{M}}(r)-J_{\mathrm{X}}(r)\right]}{\Gamma F j \omega+\frac{\left|\bar{i}_{\mathrm{M}}(r)\right|}{1-\bar{\gamma}(r)}+\frac{\left|\bar{i}_{X}(r)\right|}{\bar{\gamma}(r)}}
$$

The parameters $J_{\mathrm{M}}$ and $J_{\mathrm{X}}$ are defined to be dimensionless current densities for reactions (17) and (18), respectively, and are functions of radial position on the electrode surface, as given by

$$
J_{\mathrm{M}}(r)=\frac{b_{\mathrm{M}}\left|\bar{i}_{\mathrm{M}}(r)\right| r_{0}}{\kappa}
$$

and

$$
J_{\mathrm{X}}(r)=\frac{b_{\mathrm{X}}\left|\bar{i}_{\mathrm{X}}(r)\right| r_{0}}{\kappa}
$$

respectively. The sum of $J_{\mathrm{M}}$ and $J_{\mathrm{X}}$ represents the dimensionless current density which flows through the charge-transfer steps, i.e.,

$$
J(r)=J_{\mathrm{M}}(r)+J_{\mathrm{X}}(r)=\frac{r_{0}}{\kappa}\left[K_{\mathrm{M}} b_{\mathrm{M}}(1-\bar{\gamma}) \exp \left(b_{\mathrm{M}}\left(\bar{V}-\bar{\Phi}_{0}\right)\right)+K_{\mathrm{X}} b_{\mathrm{X}} \bar{\gamma} \exp \left(b_{\mathrm{X}}\left(\bar{V}-\bar{\Phi}_{0}\right)\right)\right]
$$

The value of $J(r)$ presented in equation (28) may be compared to the value presented in (13) for a faradaic reaction that does not involve adsorbed intermediates. 
The impedance response for coupled reactions involving an intermediate adsorbed species under potentiostatic control was given by Armstrong and Edmondson [20] and by Cao.[21] Following the method developed by Epelboin et al. [22], the faradaic impedance $Z_{F}$ at a given potential is given by

$$
\frac{1}{Z_{F}}=\frac{1}{R_{t}}+\frac{A}{j \omega+B}
$$

where the charge-transfer resistance $R_{t}$ is defined by

$$
\frac{1}{R_{t}}=\frac{1}{R_{t, \mathrm{M}}}+\frac{1}{R_{t, \mathrm{X}}}=b_{\mathrm{M}}\left|\bar{i}_{\mathrm{M}}\right|+b_{\mathrm{X}}\left|\bar{i}_{\mathrm{X}}\right|
$$

and parameters $A$ and $B$ are potential dependent variables given by

$$
A=\frac{\partial \bar{i}_{F}}{\partial \bar{\gamma}} \frac{\partial \dot{\gamma}}{\partial \bar{V}}=\frac{\left(R_{t, \mathrm{M}}^{-1}-R_{t, \mathrm{X}}^{-1}\right)\left[K_{\mathrm{X}} \exp \left(b_{\mathrm{X}}\left(\bar{V}-\bar{\Phi}_{0}\right)\right)-K_{\mathrm{M}} \exp \left(b_{\mathrm{M}}\left(\bar{V}-\bar{\Phi}_{0}\right)\right)\right]}{\Gamma F}
$$

and

$$
B=-\frac{\partial \dot{\gamma}}{\partial \bar{\gamma}}=\frac{K_{\mathrm{X}} \exp \left(b_{\mathrm{X}}\left(\bar{V}-\bar{\Phi}_{0}\right)\right)+K_{\mathrm{M}} \exp \left(b_{\mathrm{M}}\left(\bar{V}-\bar{\Phi}_{0}\right)\right)}{\Gamma F}
$$

While $B$ is always positive, the sign of $A$ varies with the potential across the electrode-electrolyte interface, and the feature of the impedance plane changes according to the sign of $A$.

When $A$ is positive, the impedance plot in the Nyquist plane of $Z_{F}$ in parallel with the double layer capacitance $\left(C_{0}\right)$ shows a high-frequency capacitive loop corresponding to $R_{t}$ in parallel with $C_{0}$, i.e., $R_{t} \| C_{0}$, with a low-frequency inductive loop corresponding to the second term of Equation (29). When $A$ is negative, the impedance plot shows a high-frequency capacitive loop $\left(R_{t} \| C_{0}\right)$ with a low-frequency capacitive loop, also corresponding to the second term of Equation (29). When $A$ is equal to zero, the two terms of the numerator in equation (31) cancel, i.e., $\partial \bar{i}_{\mathrm{F}} / \partial \bar{\gamma}=0$. In this case, the reaction current density is not dependent on the surface coverage, and, therefore, the impedance plot shows a single capacitive loop corresponding to $R_{t} \| C_{0}$ with no low-frequency loop.

\subsection{Influence of Multi-Component Transport}

A two-dimensional impedance model was developed by Wu et al. [12] to study the effect of nonuniform mass transfer for a rotating disk electrode geometry and the influence of coupled charging and faradaic currents on the impedance response.

\subsubsection{Mass Transport in Dilute Solutions}

Conservation of species accounting for mass transport in dilute solution is expressed as

$$
\frac{\partial c_{\mathrm{i}}}{\partial t}=-\nabla \cdot \mathbf{N}_{\mathrm{i}}+R_{\mathrm{i}}
$$

where the flux is given by the Nernst-Planck equation

$$
\mathbf{N}_{\mathrm{i}}=-D_{\mathrm{i}}\left(z_{\mathrm{i}} c_{\mathrm{i}} \frac{\mathrm{F}}{\mathrm{R} T} \nabla \Phi+\nabla c_{\mathrm{i}}\right)+c_{\mathrm{i}} \mathbf{v}
$$

$c_{\mathrm{i}}$ is the concentration of species $\mathrm{i}, z_{\mathrm{i}}$ is the charge number, $D_{\mathrm{i}}$ is the diffusivity, $\mathbf{v}$ is the massaveraged velocity associated with the rotating disk, and $R_{\mathrm{i}}$ represents the production of species i by 
homogeneous reactions. In the absence of homogeneous reactions, $R_{\mathrm{i}}=0$, and, under assumption that the diffusion coefficients are uniform,

$$
\frac{\partial c_{\mathrm{i}}}{\partial t}+\mathbf{v} \cdot \nabla c_{\mathrm{i}}=z_{\mathrm{i}} D_{\mathrm{i}} \frac{\mathrm{F}}{\mathrm{R} T} \nabla \cdot\left(c_{\mathrm{i}} \nabla \Phi\right)+D_{\mathrm{i}} \nabla^{2} c_{\mathrm{i}}
$$

For a system with $n$ species, $n$ expressions in the form of equation (35) are required; whereas the conservation of charge implies that

$$
\nabla \cdot \mathbf{i}=-\nabla \cdot\left(\mathrm{F} \sum_{\mathrm{i}} D_{\mathrm{i}} z_{\mathrm{i}}\left(z_{\mathrm{i}} c_{\mathrm{i}} \frac{\mathrm{F}}{\mathrm{R} T} \nabla \Phi+\nabla c_{\mathrm{i}}\right)\right)=0
$$

Equations (35) and (36) constitute a set of nonlinear differential equations.

In the frequency domain, each variable, including potential and the concentrations of each species, is described as shown in equation (2). The mass and charge conservation equations become

$$
\mathrm{j} \omega \widetilde{c}_{\mathrm{i}}+\mathbf{v} \cdot \nabla \widetilde{c}_{\mathrm{i}}=D_{\mathrm{i}} \nabla \cdot\left(\nabla \widetilde{c}_{\mathrm{i}}+\frac{z_{\mathrm{i}} \mathrm{F}}{\mathrm{RT}}\left(\bar{c}_{\mathrm{i}} \nabla \widetilde{\Phi}+\widetilde{c}_{\mathrm{i}} \nabla \bar{\Phi}\right)\right)
$$

and

$$
\nabla \cdot\left(\sum_{\mathrm{i}} z_{\mathrm{i}} D_{\mathrm{i}}\left(\nabla \widetilde{c}_{\mathrm{i}}+\frac{z_{i} \mathrm{~F}}{\mathrm{R} T}\left(\bar{c}_{\mathrm{i}} \nabla \widetilde{\Phi}+\widetilde{c}_{\mathrm{i}} \nabla \bar{\Phi}\right)\right)\right)=0
$$

respectively, where the higher order terms such as $\widetilde{c}_{\mathrm{i}} \nabla \widetilde{\Phi}$ were neglected. At the electrode surface, the flux of each species may be expressed as

$$
\widetilde{N}_{\mathrm{i}, \mathrm{y}}(0)=-D_{\mathrm{i}}\left(\left.\frac{\mathrm{d} \widetilde{c}_{\mathrm{i}}}{\mathrm{d} y}\right|_{y=0}+\frac{z_{\mathrm{i}} \mathrm{F}}{\mathrm{R} T}\left(\left.\bar{c}_{\mathrm{i}} \frac{\mathrm{d} \widetilde{\Phi}}{\mathrm{d} y}\right|_{y=0}+\left.\widetilde{c}_{\mathrm{i}} \frac{\mathrm{d} \bar{\Phi}}{\mathrm{d} y}\right|_{y=0}\right)\right)
$$

The correlation between the flux and the current oscillations at electrode boundary is discussed in two cases where the faradaic current and the double-layer charging current are considered with and without a priori separation of faradaic and charging currents.

\subsubsection{No a Priori Separation of Faradaic and Charging Currents (NAPS)}

Under the assumption that the charge density on the metal surface $q_{\mathrm{m}}$ is dependent on the interfacial potential $V$ and on the concentration $c_{\mathrm{i}}(0)$ of each species i, located outside the diffuse region of charge, the variation of the surface charge density is given by

$$
\mathrm{d} q_{\mathrm{m}}=\left(\frac{\partial q_{\mathrm{m}}}{\partial V}\right)_{c_{\mathrm{i}}(0)} \mathrm{d} V+\sum_{\mathrm{i}}\left(\frac{\partial q_{\mathrm{m}}}{\partial c_{\mathrm{i}}(0)}\right)_{V, c_{\mathrm{j} \neq \mathrm{i}}(0)} \mathrm{d} c_{\mathrm{i}}(0)
$$

Equation (40) is written in terms of $n+1$ parameters which are treated as properties of the interface. These can be expressed as

$$
C_{0}=\left(\frac{\partial q_{\mathrm{m}}}{\partial V}\right)_{c_{\mathrm{i}}(0)}
$$

where $C_{0}$ is the usual differential capacitance, and

$$
C_{\mathrm{i}}=\left(\frac{\partial q_{\mathrm{m}}}{\partial c_{\mathrm{i}}(0)}\right)_{V, c_{\mathrm{j} \neq \mathrm{i}}(0)}
$$


which can be expressed for species $\mathrm{i}=1, \ldots, n$. Both terms may be be obtained from detailed models of the diffuse double layer.

The current at the electrode surface may be expressed as

$$
\widetilde{i}=\mathrm{j} \omega \widetilde{q}_{\mathrm{m}}+\widetilde{i}_{\mathrm{F}}
$$

where the oscillations of the surface charge density and the faradaic current density are approximated by Taylor series expansions about their steady values as

$$
\widetilde{q}_{\mathrm{m}}=\left(\frac{\partial \bar{q}_{\mathrm{m}}}{\partial V}\right)_{c_{\mathrm{i}}(0)} \widetilde{V}+\sum_{\mathrm{i}}\left(\frac{\partial \bar{q}_{\mathrm{m}}}{\partial c_{\mathrm{i}}(0)}\right)_{V, c_{\mathrm{j} \neq \mathrm{i}}(0)} \widetilde{c}_{\mathrm{i}}(0)
$$

and

$$
\widetilde{i}_{\mathrm{F}}=\left(\frac{\partial \bar{i}_{\mathrm{F}}}{\partial V}\right)_{c_{\mathrm{i}}(0)} \widetilde{V}+\sum_{\mathrm{i}}\left(\frac{\partial \bar{i}_{\mathrm{F}}}{\partial c_{\mathrm{i}}(0)}\right)_{V, c_{\mathrm{j} \neq \mathrm{i}}(0)} \widetilde{c}_{\mathrm{i}}(0)
$$

respectively. The surface flux can be expressed as

$$
\widetilde{N}_{\mathrm{i}, \mathrm{y}}(0)=-\frac{\partial \Gamma_{\mathrm{i}}}{\partial c_{\mathrm{i}}(0)} \frac{\partial c_{\mathrm{i}}(0)}{\partial q_{\mathrm{m}}} \mathrm{j} \omega \widetilde{q}_{\mathrm{m}}-\frac{s_{\mathrm{i}}}{n \mathrm{~F}} \widetilde{i}_{\mathrm{F}}
$$

where $s_{\mathrm{i}}$ is the stoichiometric coefficient for the reaction and $\Gamma_{\mathrm{i}}$ is the surface concentration of species i. Equations (43) and (46) were applied as the boundary conditions to evaluate the impedance response without a priori assumption of the separation of faradaic and charging currents.

\subsubsection{Double-Layer Model}

The Gouy-Chapman-Stern model was used by Wu et al. [12] to describe the electrical behavior in the diffuse double layer. Under the assumption that ion-specific adsorption could be neglected, values for $C_{0}$ and $C_{\mathrm{i}}$, equations (41) and (42), respectively, were obtained as functions of potential and the concentration of individual ionic species at the outer limit of the diffuse part of the double layer. The structure of the electrical double layer is illustrated in Figure 1 where the metal layer $(\mathrm{M})$ is associated with the excess charge of electrons at the metal surface, the outer Helmholtz plane (OHP) is the plane of closest approach for solvated ions and the inner Helmholtz plane (IHP) is the plane associated with ions adsorbed to the electrode.

As the thickness of the double layer is very thin, it is often considered to be a part of the electrode-electrolyte interface. The interfacial region as a whole obeys electrical neutrality such that the excess surface charge density at electrode is balanced by the surface charges at the IHP and in the diffuse part of the double layer, i.e.,

$$
q_{\mathrm{m}}+q_{\mathrm{ihp}}+q_{\mathrm{d}}=0
$$

The surface charge density is related to the surface excess concentration of charged species by

$$
q_{\mathrm{m}}=-\left(q_{\mathrm{ihp}}+q_{\mathrm{d}}\right)=-\mathrm{F} \sum_{\mathrm{i}} z_{\mathrm{i}} \Gamma_{\mathrm{i}}
$$

The mean electrostatic potentials at the metal surface, the IHP, and the OHP are denoted by $\Phi_{\mathrm{m}}$, $\Phi_{\text {ihp }}$, and $\Phi_{\text {ohp }}$, respectively. The surface concentrations $c_{\mathrm{i}}(0)$ and potential $\Phi_{0}$ used in the rate 


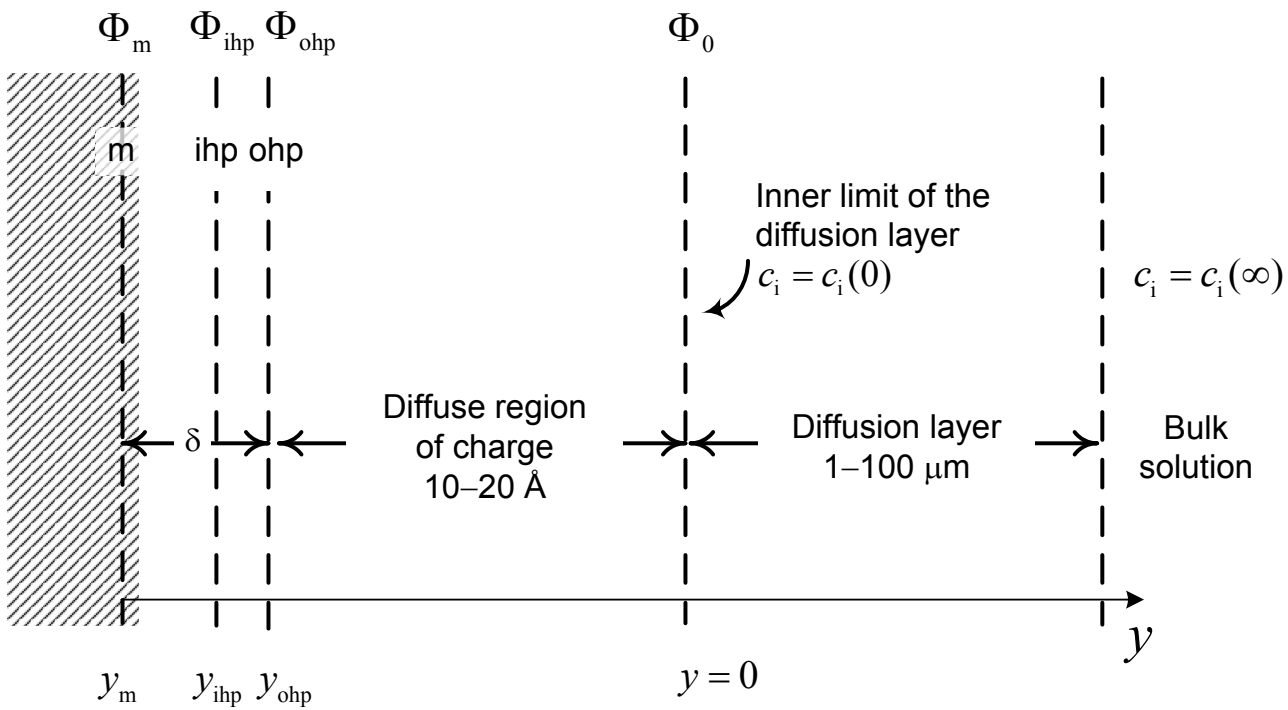

Figure 1: Schematic representation of the electrical double layer structure following a Stern-Gouy-Chapman model. The sketch is not drawn to scale. Taken from Wu et al. [12]

expressions are usually evaluated at the outer limit of the diffuse layer, or the inner limit of the diffusion layer.

The ionic concentrations in the diffuse part of the double layer are assumed to have a Boltzmann distribution, i.e.,

$$
c_{\mathrm{i}}=c_{\mathrm{i}}(\infty) \exp \left(-\frac{z_{\mathrm{i}} \mathrm{F} \Phi}{\mathrm{R} T}\right)
$$

Poisson's equation provides the relationship between the concentrations and potential as

$$
\frac{\mathrm{d}^{2} \Phi}{\mathrm{d} y^{2}}=-\frac{\mathrm{F}}{\epsilon_{\mathrm{d}} \epsilon_{0}} \sum_{\mathrm{i}} z_{\mathrm{i}} c_{\mathrm{i}}=-\frac{\mathrm{F}}{\epsilon_{\mathrm{d}} \epsilon_{0}} \sum_{\mathrm{i}} z_{\mathrm{i}} c_{\mathrm{i}}(\infty) \exp \left(-\frac{z_{\mathrm{i}} \mathrm{F} \Phi}{\mathrm{R} T}\right)
$$

where $y$ is the distance from the electrode, $\epsilon_{\mathrm{d}}$ is the dielectric constant in the diffuse layer, and $\epsilon_{0}$ is the permittivity of vacuum $\left(\epsilon_{0}=8.8542 \times 10^{-14} \mathrm{~F} / \mathrm{cm}\right)$. Integration of the Poisson equation gives the relation of potential gradient to the surface charge density in the diffuse layer as

$$
\left.\frac{\mathrm{d} \Phi}{\mathrm{d} y}\right|_{y_{\mathrm{ohp}}}=\frac{q_{\mathrm{d}}}{\epsilon_{\mathrm{d}} \epsilon_{0}}
$$

where $y_{\text {ohp }}$ is located at the inner limit of the diffuse part of the double layer, as indicated in Figure 1. At equilibrium, the potential approaches zero far from the electrode surface, and Poisson's equation can then be solved by applying the boundary conditions to yield the charge density in the diffuse layer, i.e.,

$$
q_{\mathrm{d}}=\mp\left\{2 \mathrm{R} T \epsilon_{\mathrm{d}} \epsilon_{0} \sum_{i} c_{\mathrm{i}}(\infty)\left[\exp \left(\frac{-z_{\mathrm{i}} \mathrm{F} \Phi_{\mathrm{ohp}}}{\mathrm{R} T}\right)-1\right]\right\}^{1 / 2}
$$


The upper sign is used if the potential is positive and, conversely, the lower sign is used if the potential is negative.

In the absence of ion-specific adsorption on the IHP, i.e., $q_{\mathrm{ihp}}=0$, the surface concentration is related to the individual charge density within the diffuse part of the double layer by

$$
\Gamma_{\mathrm{i}}=\frac{q_{\mathrm{d}, \mathrm{i}}}{z_{\mathrm{i}} \mathrm{F}}
$$

Following the convention adopted by Gibbs (see for example, Chapter 7 in reference [23]) the surface charge associated with a given species i within the diffuse part of the double layer can be expressed as

$$
q_{\mathrm{d}, \mathrm{i}}=z_{\mathrm{i}} \mathrm{F} \int_{y_{\mathrm{ohp}}}^{\infty}\left(c_{\mathrm{i}}-c_{\mathrm{i}}(\infty)\right) \mathrm{d} y
$$

where $c_{\mathrm{i}}$ is given by equation (49). Integration over $y$ is accomplished by use of the definition of electric field, i.e.,

$$
E=-\frac{\mathrm{d} \Phi}{\mathrm{d} y}
$$

where $E$ is obtained as a function of $\Phi$ from equations (51) and (52). The charge density associated with individual species is therefore obtained by an integral over the potential drop in the diffuse region as

$$
q_{\mathrm{d}, \mathrm{i}}=\mp \int_{0}^{\Phi_{\mathrm{ohp}}} \frac{z_{\mathrm{i}} \mathrm{F} c_{\mathrm{i}}(\infty)\left[\exp \left(\frac{-z_{\mathrm{i}} \mathrm{F} \Phi}{\mathrm{R} T}\right)-1\right]}{\left\{\frac{2 \mathrm{R} T}{\epsilon_{\mathrm{d}} \epsilon_{0}} \sum_{\mathrm{k}} c_{\mathrm{k}}(\infty)\left[\exp \left(\frac{-z_{\mathrm{k}} \mathrm{F} \Phi}{\mathrm{R} T}\right)-1\right]\right\}^{1 / 2}} \mathrm{~d} \Phi
$$

The above expressions for charge densities were derived by assuming a true equilibrium of the system in which the ionic concentrations at the outer limit of the diffuse layer are the same as the bulk values, i.e., $c_{\mathrm{i}}(0)=c_{\mathrm{i}}(\infty)$, and the potential at the outer limit of the diffuse layer is equal to that of a reference electrode placed at infinity; thus, for a reference electrode of the same kind as the working electrode, $\Phi_{0}=0$.

When the net current flowing to the electrode is not equal to zero, the system is not at equilibrium, i.e., $\Phi_{0} \neq 0$. Equations (52) and (56) become

$$
q_{\mathrm{d}}=\mp\left\{2 \mathrm{R} T \epsilon_{\mathrm{d}} \epsilon_{0} \sum_{\mathrm{i}} c_{\mathrm{i}}(0)\left[\exp \left(\frac{-z_{\mathrm{i}} \mathrm{F}\left(\Phi_{\mathrm{ohp}}-\Phi_{0}\right)}{\mathrm{R} T}\right)-1\right]\right\}^{1 / 2}
$$

and

$$
q_{\mathrm{d}, \mathrm{i}}=\mp \int_{0}^{\Phi_{\mathrm{ohp}}} \frac{z_{\mathrm{i}} \mathrm{F} c_{\mathrm{i}}(0)\left[\exp \left(\frac{-z_{\mathrm{i}} \mathrm{F} \Phi}{\mathrm{R} T}\right)-1\right]}{\left\{\frac{2 \mathrm{R} T}{\epsilon_{\mathrm{d}} \epsilon_{0}} \sum_{\mathrm{k}} c_{\mathrm{k}}(0)\left[\exp \left(\frac{-z_{\mathrm{k}} \mathrm{F} \Phi}{\mathrm{R} T}\right)-1\right]\right\}^{1 / 2}} \mathrm{~d} \Phi
$$

respectively. The charge densities in the diffuse layer are now related to the concentrations and potential at the outer limit of the diffuse layer, which can be obtained by solving the mass and charge conservation equations outside the diffuse region of charge. 
The evaluation of the surface charge density in equation (52) or (57) requires additional information in the electrical double layer. Gauss's law relates the surface charge density to the electric field within the OHP by

$$
q_{\mathrm{m}}=-q_{\mathrm{d}}=\frac{\epsilon \epsilon_{0}}{\delta}\left(\Phi_{\mathrm{m}}-\Phi_{\mathrm{ohp}}\right)
$$

where $\epsilon$ is the dielectric constant between the metal surface and the OHP and $\delta$ is the distance between the metal surface and the OHP, as shown in Figure 1. Equation (59) can be used as a second equation to solve for $q_{\mathrm{m}}$ and $\Phi_{\mathrm{ohp}}$ in the electrical double layer.

\subsubsection{With a Priori Separation of Faradaic and Charging Currents (APS)}

The conventional approach is to consider the double-layer charging and the faradaic currents to be separable quantities, as is done in most of published works (see, e.g., reference [24]). The total current density is expressed by

$$
i=i_{\mathrm{C}}+i_{\mathrm{F}}=C_{0} \frac{\mathrm{d} V}{\mathrm{~d} t}+i_{\mathrm{F}}
$$

or, in frequency domain,

$$
\widetilde{i}=\mathrm{j} \omega C_{0} \widetilde{V}+\widetilde{i}_{\mathrm{F}}=\mathrm{j} \omega\left(\frac{\partial q_{\mathrm{m}}}{\partial V}\right)_{c_{\mathrm{i}}(0)} \widetilde{V}+\widetilde{i}_{\mathrm{F}}
$$

The contribution of mass flux in charging the double layer is neglected when the faradaic current and the charging current are considered separately. Thus,

$$
\widetilde{N}_{\mathrm{i}, \mathrm{y}}(0)=-\frac{s_{\mathrm{i}}}{n \mathrm{~F}} \widetilde{i}_{\mathrm{F}}
$$

Comparison of equation (62) with equation (46) shows that the first term in equation (46) is neglected. Under the assumption that there is a priori separation of faradaic and charging currents, equations (61) and (62) are used as the boundary conditions to evaluate the transient responses of potential and concentrations.

\section{Numerical Method}

Solutions for the two classifications of disk impedance models require difference numerical approaches.

\subsection{Influence of Ohmic and Kinetic Phenomena}

Two methods were used to solve the sets of equations. In one method, following Newman, [6] Laplace's equation was transformed to rotational elliptic coordinates. The equations were solved under assumption of a uniform capacitance $C_{0}$ using the collocation package PDE2D ${ }^{\circledR}$ developed by Sewell. [25] Calculations were performed for differing domain sizes, and the results were obtained by extrapolation to an infinite domain size.

The equations were also solved in the cylindrical coordinates using a finite-elements package COMSOL. ${ }^{\circledR}$ The domain size was 2,000 times larger than the disk electrode dimension in order to meet the assumption that the counterelectrode was located infinitely far from the electrode surface. The results obtained by the two packages were in excellent agreement for dimensionless frequencies $\mathrm{K}<100$, providing a frequency domain covering the entire electrochemical response of the system. 


\subsection{Influence of Multi-Component Transport}

Numerical methods were used to solve the sets of equations corresponding to the steady-state distributions of concentration and the model for the diffuse double layer. These provided the parameters presented in equations (41) and (42) that were needed for the numerical solution of the coupled convective diffusion equations in frequency domain.

\subsubsection{Steady-State Calculations}

Equations (35) and (36) were solved under the assumption of a steady state using the finiteelement package COMSOL Multiphysics ${ }^{\circledR}$ with the Nernst-Planck module in a 2D axial symmetric coordinate system. The domain size was identical to that used for the impedance calculations presented in section 3.2.3. The values for the concentrations at the inner limit of the diffusion layer were used as input for the double-layer model described in section 3.2.2, and, as the problem is nonlinear due to the explicit treatment of the migration term, the steady-state values of potential and concentration were used for the impedance calculations described in section 3.2.3.

\subsubsection{Double-Layer Properties}

The nonlinear equations relating to the charge and potential distributions in the electrical double layer were solved by using the Newton-Raphson method. For nonequilibrium systems, a local equilibrium was assumed in which the concentrations and potential at the outer limit of the diffuse layer were given by the steady-state solution of the convective diffusion equations. The radially dependent surface concentrations and potential gave a radial distribution of charge over the electrode surface. The surface-averaged value of charge was obtained from the surface-averaged values of concentration and potential. The charge in the diffuse layer associated with individual species was obtained by subsequent numerical integration of equation (56) or (58), using the MATLAB integration function, the adaptive Gauss-Kronrod quadrature method. The thickness of the compact layer between the metal surface and the OHP was assumed to be $3 \AA$. The dielectric constant in the compact region within the OHP was approximately 6 , according to Bockris $[26]$ for a fully oriented water layer next to the electrode surface. The dielectric constant in the diffuse layer was assumed to be 78 , which is the value of water at room temperature.

\subsubsection{Impedance Calculations}

The equations were solved for a rotating disk electrode by using the finite-element package COMSOL Multiphysics ${ }^{\circledR}$ with the Nernst-Planck module in a 2D axial symmetric coordinate system. The domain size was 2,000 times larger than the disk radius in order to meet the assumption that the counterelectrode was located infinitely far from the electrode surface. The meshed domain used to calculate the coupled solution for potential and concentrations is shown in Figure 2. A coarse mesh was used for the domain distant from the electrode to reduce physical memory usage and calculation time. A finer mesh was applied in the region that is 20 times larger than the disk radius to capture the variation of potential in the vicinity of the electrode. Since the concentration of ionic species varies only in a small distance above the electrode surface, a much finer mesh was constructed in the region that is ten times larger than the characteristic thickness of the diffusion layer

$$
\delta_{\mathrm{N}}=\left(\frac{3 D_{\mathrm{i}}}{a \nu}\right)^{1 / 3}\left(\frac{\nu}{\Omega}\right)^{1 / 2}
$$




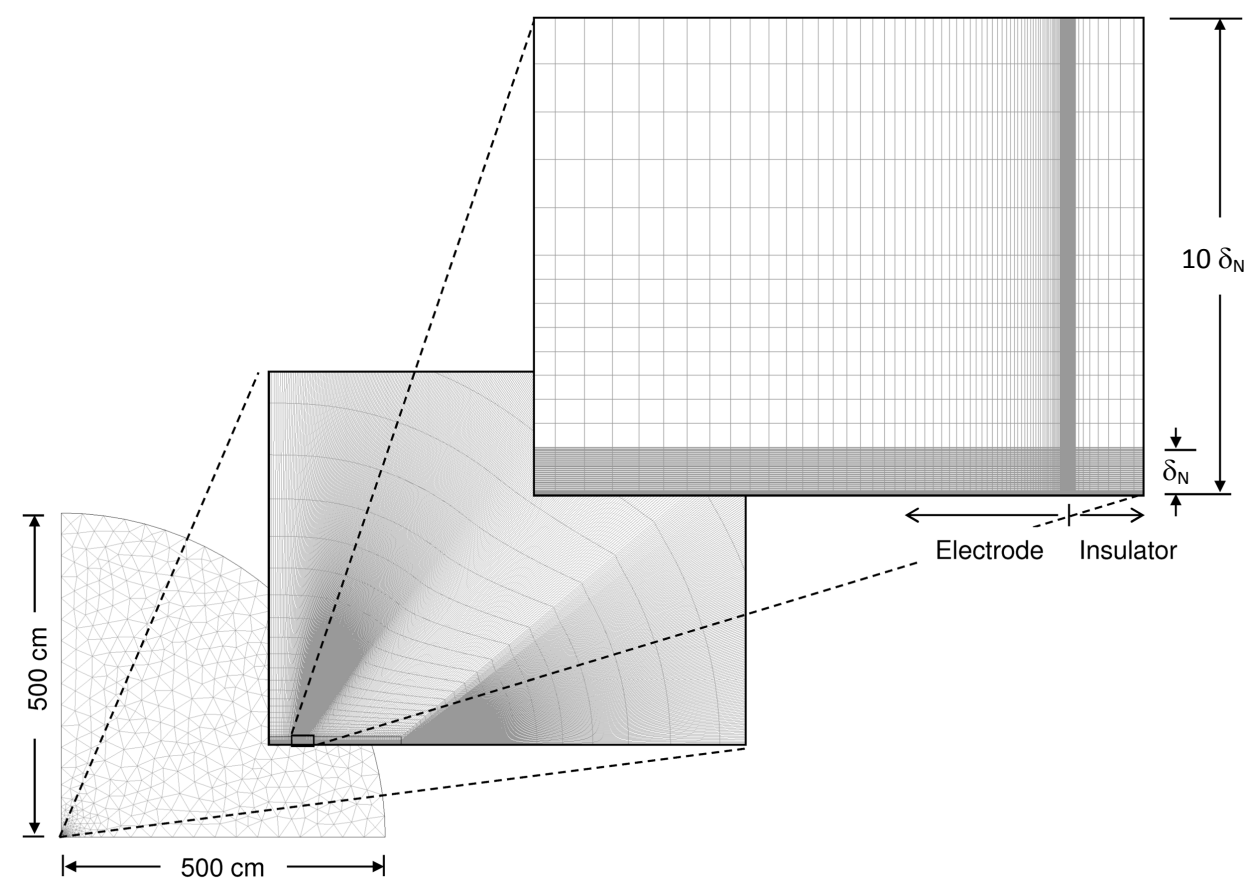

Figure 2: Representation of the different mesh scales used for the finite-element calculations. Taken from We et al. [12] 
where $a=0.51023$ is the coefficient for the first term in the velocity expansion for the rotating disk, $\nu$ is the kinematic viscosity, and $\Omega$ is the disk rotation speed.[23] This approach captured the concentration variation at the electrode surface.

Steady-state solutions were used in the impedance model in the form of lookup tables from which appropriate values could be obtained by interpolation. The use of the same meshed domain in the steady-state and the impedance models reduced the error from interpolation between nodal points. The distributions of the double-layer capacitance and other thermodynamic parameters at electrode boundary were applied in the impedance model to evaluate the impedance response for the NAPS case. For the APS case, a surface-averaged double-layer capacitance

$$
\left\langle C_{0}\right\rangle=\frac{1}{\pi r_{0}^{2}} \int_{0}^{r_{0}} C_{0}(r) r \mathrm{~d} r
$$

was used to determine the charging current that did not include the contribution of mass transfer in the diffuse region of charge.

The velocity used in the expression for convective diffusion consisted of an interpolation formula that followed the three-term velocity expansion for radial and axial velocity components appropriate for the velocity boundary layer near the disk electrode surface and the two-term expansion appropriate for the region far from the disk surface (see Cochran[27]). The interpolation formula provided excellent accuracy near the electrode surface and showed good agreement with numerical solutions of the Navier-Stokes equations throughout the domain.

\section{Results and Discussion}

Some key results of the simulations are presented here in terms of global impedance. While local impedance may be measured and is useful for exploring detailed electrode response, the global impedance corresponds to the much more commonly used impedance measurement.

\subsection{Influence of Ohmic and Kinetic Phenomena}

One result of the simulations based on use of Laplace's equation to account for potential in the electrolyte was that the ohmic contribution has a complex character. This may be seen at high frequencies for systems that do not involve an adsorbed reaction intermediate. For systems that involve an adsorbed reaction intermediate, frequency dispersion may be seen at both high and low frequencies.

\subsubsection{Complex Ohmic Impedance at High Frequencies}

The global impedance response, calculated under assumption of Tafel kinetics, is presented in Figure 3 as a function of dimensionless frequency with $J$ as a parameter. The value $J=0$ corresponds to an ideally capacitive blocking electrode. At low frequencies, values for the real part of the impedance differ for impedance calculated under the assumptions of linear and Tafel kinetics, whereas, the values of the imaginary impedance calculated under the assumptions of linear and Tafel kinetics are superposed for all frequencies. The slopes of the lines presented in Figure 3(b) are equal to +1 at low frequencies but differ from -1 at high frequencies. The slope of these lines in the high-frequency range can be related to the exponent $\alpha$ used in models for CPE behavior.[28] 


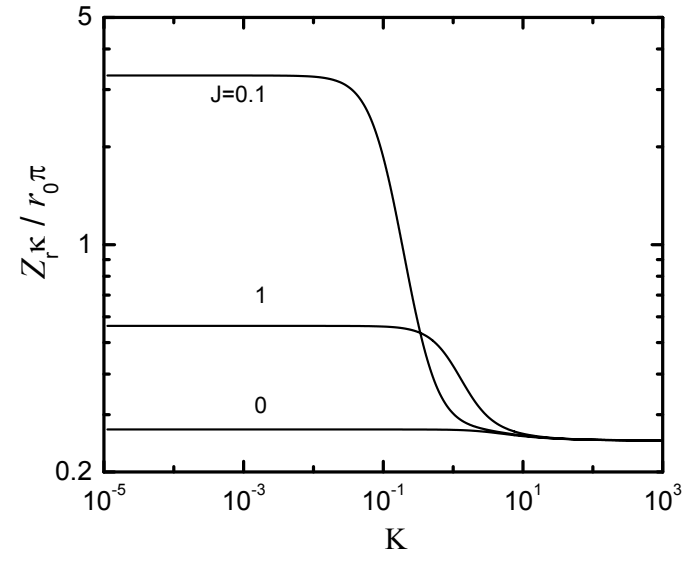

(a)

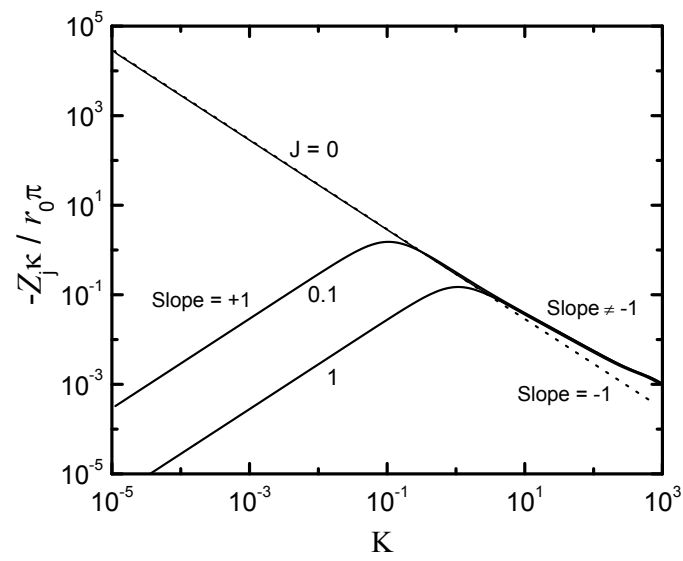

(b)

Figure 3: Calculated representation of the impedance response for a disk electrode under assumption of Tafel kinetics with $J$ as a parameter: a) real part; and b) imaginary part. The value $J=0$ corresponds to an ideally capacitive blocking electrode.

Two characteristic frequencies are evident in Figure 3. The characteristic frequency $\mathrm{K}=1$ is associated with the influence of current and potential distributions and can be expressed in terms of the capacitance $C_{0}$ and the ohmic resistance to a disk electrode[1]

$$
R_{\mathrm{e}}=\frac{\pi r_{0}}{4 \kappa}
$$

as

$$
K=\frac{4}{\pi} \omega R_{\mathrm{e}} C_{0}
$$

The characteristic frequency $\mathrm{K} / J=1$ is associated with the $R_{\mathrm{t}} C_{0}$-time constant for the faradaic reaction.

The frequency $\mathrm{K}=1$ at which the current and potential distributions begin to influence the impedance response can be expressed as

$$
f=\frac{\kappa}{2 \pi C_{0} r_{0}}
$$

or, in terms of electrolyte resistance, as

$$
f=\frac{1}{8 C_{0} R_{\mathrm{e}}}
$$

The global interfacial impedance for linear kinetics is independent of radial position and is given by

$$
Z_{0}=\frac{R_{\mathrm{t}}}{1+j \omega C_{0} R_{\mathrm{t}}}
$$

The global ohmic impedance $Z_{\mathrm{e}}$ is obtained from the global impedance $Z$ by the expression

$$
Z_{\mathrm{e}}=Z-Z_{0}
$$




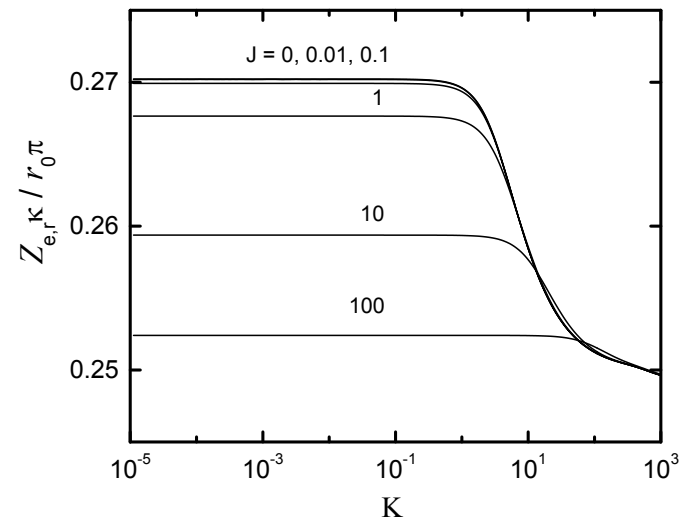

(a)

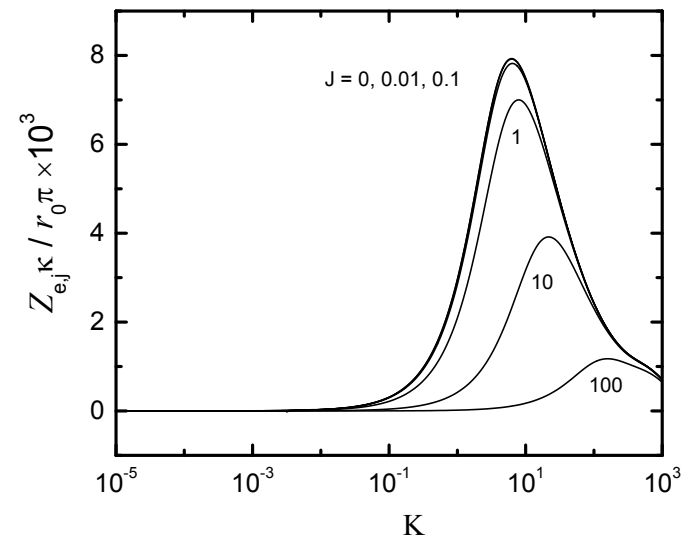

(b)

Figure 4: Calculated global ohmic impedance as a function of dimensionless frequency with $J$ as a parameter: a) real part; and b) imaginary part.

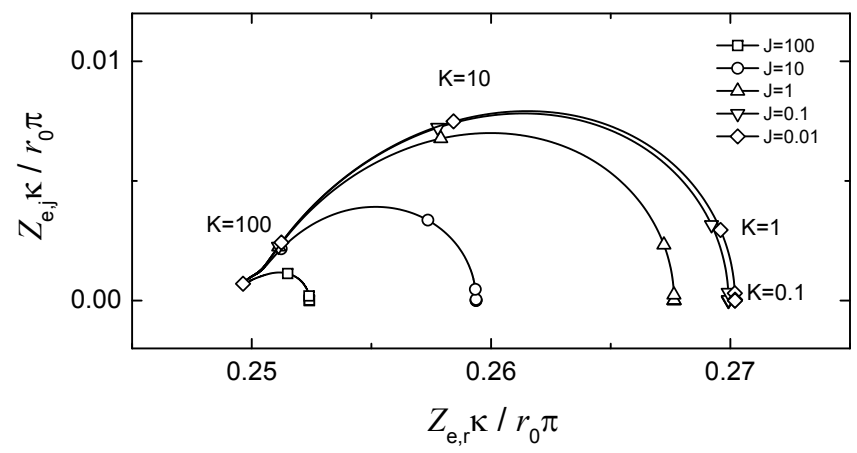

Figure 5: Calculated global ohmic impedance taken from Figure 4 in Nyquist format.

The real and imaginary parts of $Z_{\mathrm{e}}$, obtained for linear kinetics, are given in Figures $4(\mathrm{a})$ and $4(\mathrm{~b})$, respectively, as functions of dimensionless frequency $\mathrm{K}$ with $J$ as a parameter. In the low-frequency range $Z_{\mathrm{e}} \kappa / r_{0} \pi$ is a pure resistance with a numerical value that depends weakly on $J$. All curves converge in the high-frequency range such that $Z_{e} \kappa / r_{0} \pi$ tends toward $1 / 4$. The imaginary part of the global ohmic impedance shows a non-zero value in the frequency range that is influenced by the current and potential distributions. The global ohmic impedance is presented in Nyquist format in Figure 5. For $K<0.1$, the ohmic impedance is a real number, as shown in Figure 4(a). At frequencies $K>0.1$, the ohmic contribution is represented by a complex number.

The influence of high-frequency geometry-induced time-constant dispersion can be avoided for reactions that do not involve adsorbed intermediates by conducting experiments below the characteristic frequency given in equation (67). The characteristic frequency can be well within the range of experimental measurements. The value $\kappa / C_{0}=10^{3} \mathrm{~cm} / \mathrm{s}$, for example, can be obtained for a capacitance $C_{0}=10 \mu \mathrm{F} / \mathrm{cm}^{2}$ (corresponding to the value expected for the double layer on 
a metal electrode) and conductivity $\kappa=0.01 \mathrm{~S} / \mathrm{cm}$ (corresponding roughly to a $0.1 \mathrm{M} \mathrm{NaCl}$ solution). Equation (67) suggests that time-constant dispersion should be expected above a frequency of $600 \mathrm{~Hz}$ on a disk with radius $r_{0}=0.25 \mathrm{~cm}$.

The concept of a complex ohmic impedance is in agreement with Newmans calculation of frequency dispersion. A blocking interface with a frequency-independent capacitance $C_{0}$ has an interfacial impedance $Z_{0}=1 / \mathrm{j} \omega C_{0}$. This interfacial impedance is independent of the electrode geometry. The measured overall impedance, which includes the ohmic contribution, is $Z=Z_{\mathrm{e}}+1 / \mathrm{j} \omega C_{0}$, where the capacitance $C_{0}$ is independent of frequency and $Z_{\mathrm{e}}$ is termed the ohmic impedance. Newman, in contrast, represented the overall impedance as the sum of a frequency-dependent resistance $R_{\text {eff }}$ in series with a frequency-dependent capacitance $C_{\text {eff }}$. The two descriptions of the same phenomena give

or

$$
Z_{e}+\frac{1}{\mathrm{j} \omega C_{0}}=R_{\mathrm{eff}}+\frac{1}{\mathrm{j} \omega C_{\mathrm{eff}}}
$$

$$
Z_{e}=R_{\mathrm{eff}}-\frac{\mathrm{j}}{\omega}\left(\frac{C_{0}-C_{\mathrm{eff}}}{C_{0} C_{\mathrm{eff}}}\right)
$$

The observation that $Z_{\mathrm{e}}$ is frequency dependent is in perfect agreement with Newmans result.[6] When the frequency tends towards infinity, the current distribution corresponds to the primary current distribution and,

$$
\lim _{\omega \rightarrow \infty} Z_{e}=\frac{1}{4 \kappa r_{0}}
$$

in agreement with Newmans formula. The complex character of the ohmic impedance is a property of the electrode geometry, interfacial impedance, and electrolyte with conductivity $\kappa$. A complex ohmic impedance, for example, is not seen for recessed electrodes for which both the current density and interfacial potential are uniform.

\subsubsection{Complex Ohmic Impedance at High and Low Frequencies}

The results presented here are based on simulations presented by Wu et al. [10] A steady-state polarization curve is presented in Figure 6(a) where dashed boxes are used to represent the range of steady-state current density and potential corresponding to the three cases shown in Figure 7. The current distribution becomes more nonuniform at larger values of potential. For $\langle A\rangle=-0.83$ $\Omega^{-1} \mathrm{~cm}^{-2} \mathrm{~s}^{-1}$, the parameter $J$ has the largest value, meaning, as shown in equation (16), that the ohmic resistance is much larger than the charge-transfer resistance. The corresponding adsorption isotherm given in Figure 6(b) shows an inflection at $\langle A\rangle=0$, representing the stronger dependence of the surface coverage on the interfacial potential; whereas, at $\langle A\rangle=-0.83 \Omega^{-1} \mathrm{~cm}^{-2} \mathrm{~s}^{-1}$, the isotherm crosses the largest potential interval, indicating the most nonuniform potential distribution on the electrode surface.

The global impedance represents an averaged response of the electrode. The calculated results of global impedance for the cases with $\langle A\rangle>0\left(0.011 \Omega^{-1} \mathrm{~cm}^{-2} \mathrm{~s}^{-1}\right),\langle A\rangle=0$, and $\langle A\rangle<0(-0.83$ $\Omega^{-1} \mathrm{~cm}^{-2} \mathrm{~s}^{-1}$ ) are presented in Nyquist format in Figures $7(\mathrm{a}), 7(\mathrm{~b})$, and $7(\mathrm{c})$, respectively. The solid lines in Figure 7 represent the simulation results by solving the Laplace's equation coupling the boundary conditions that account for the time-constant dispersion associated with the electrode geometry. The dashed curves represent the global impedances calculated by

$$
Z=R_{e}+\frac{1}{1 / Z_{\mathrm{F}}+j \omega C_{0}}
$$




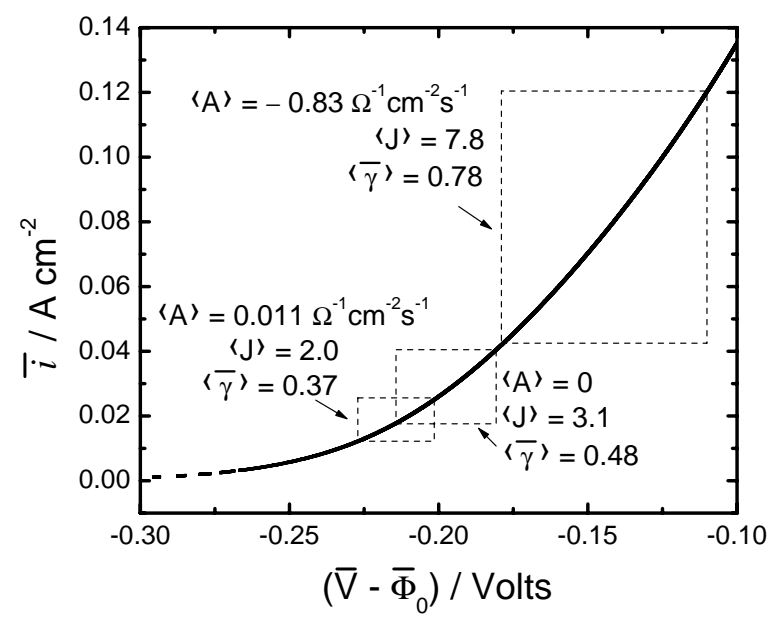

(a)

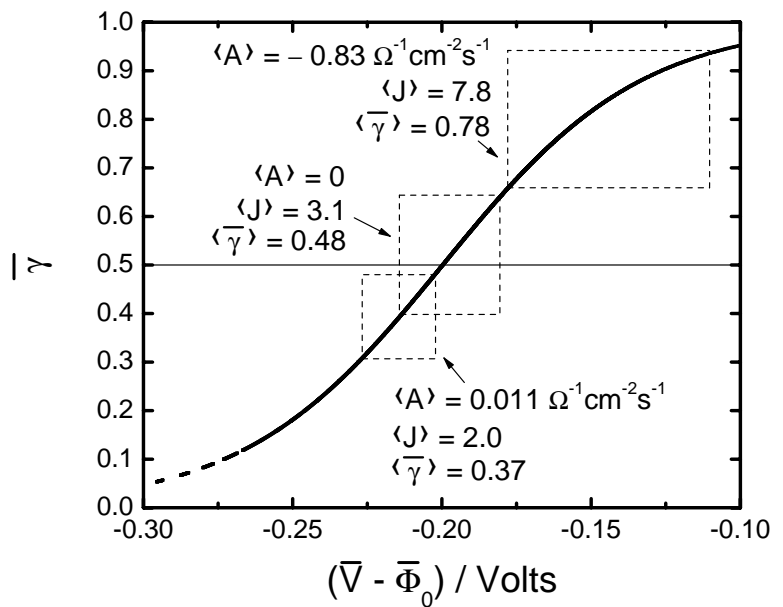

(b)

Figure 6: The variations of a) steady-state current density and b) steady-state surface coverage density with the interfacial potential. Dashed squares are used to identify the range of current and surface coverage corresponding to the simulations performed at $\bar{V}=-0.15 \mathrm{~V}\left(\langle A\rangle=0.011 \Omega^{-1} \mathrm{~cm}^{-2} \mathrm{~s}^{-1}\right), \bar{V}=-0.1 \mathrm{~V}\left(\langle A\rangle=0 \Omega^{-1} \mathrm{~cm}^{-2} \mathrm{~s}^{-1}\right)$, and $\bar{V}=0.1 \mathrm{~V}\left(\langle A\rangle=-0.83 \Omega^{-1} \mathrm{~cm}^{-2} \mathrm{~s}^{-1}\right)$. The position $r=0$ corresponds to the lower-left corner of each box. Taken from Wu et al. [10] 


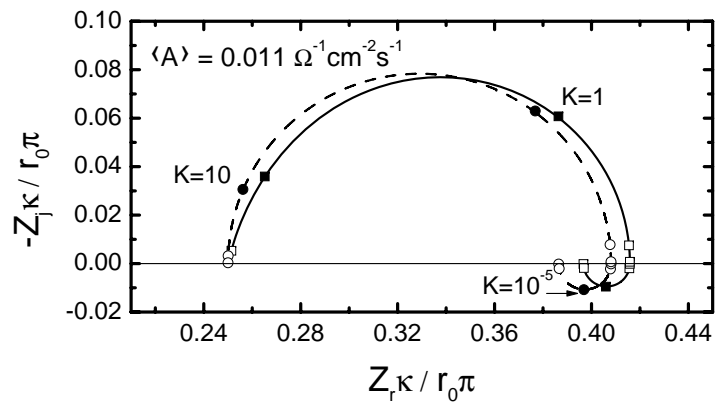

(a)

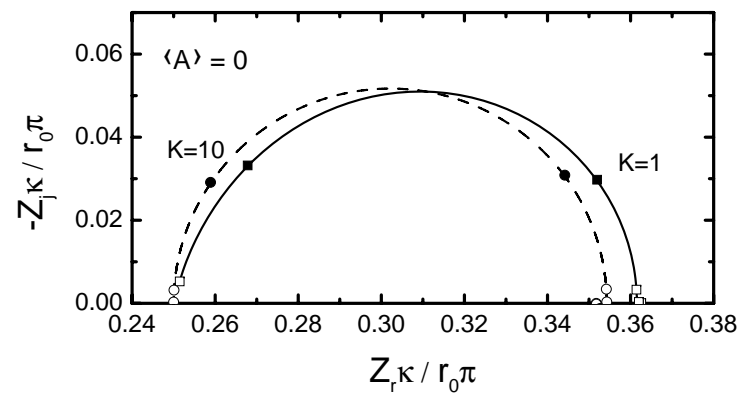

(b)

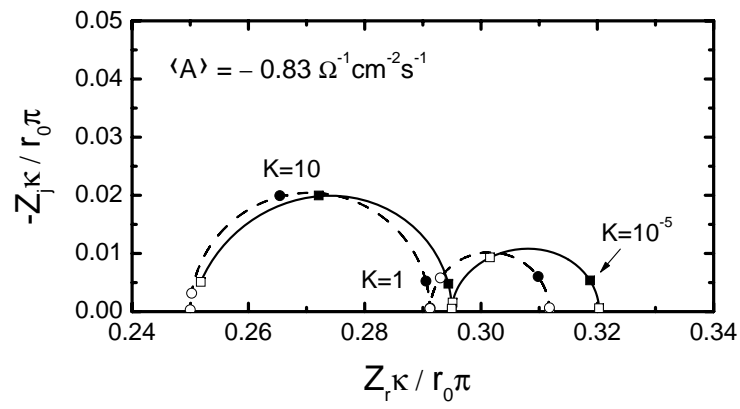

(c)

Figure 7: Calculated Nyquist representation of the global impedance response for a disk electrode considering the influence of electrode geometry (solid lines) and in the absence of geometry effect (dashed lines): a) $\langle A\rangle>0$; b) $\langle A\rangle=0$; and $(\mathrm{c})\langle A\rangle<0$. Taken from Wu et al. [10] 


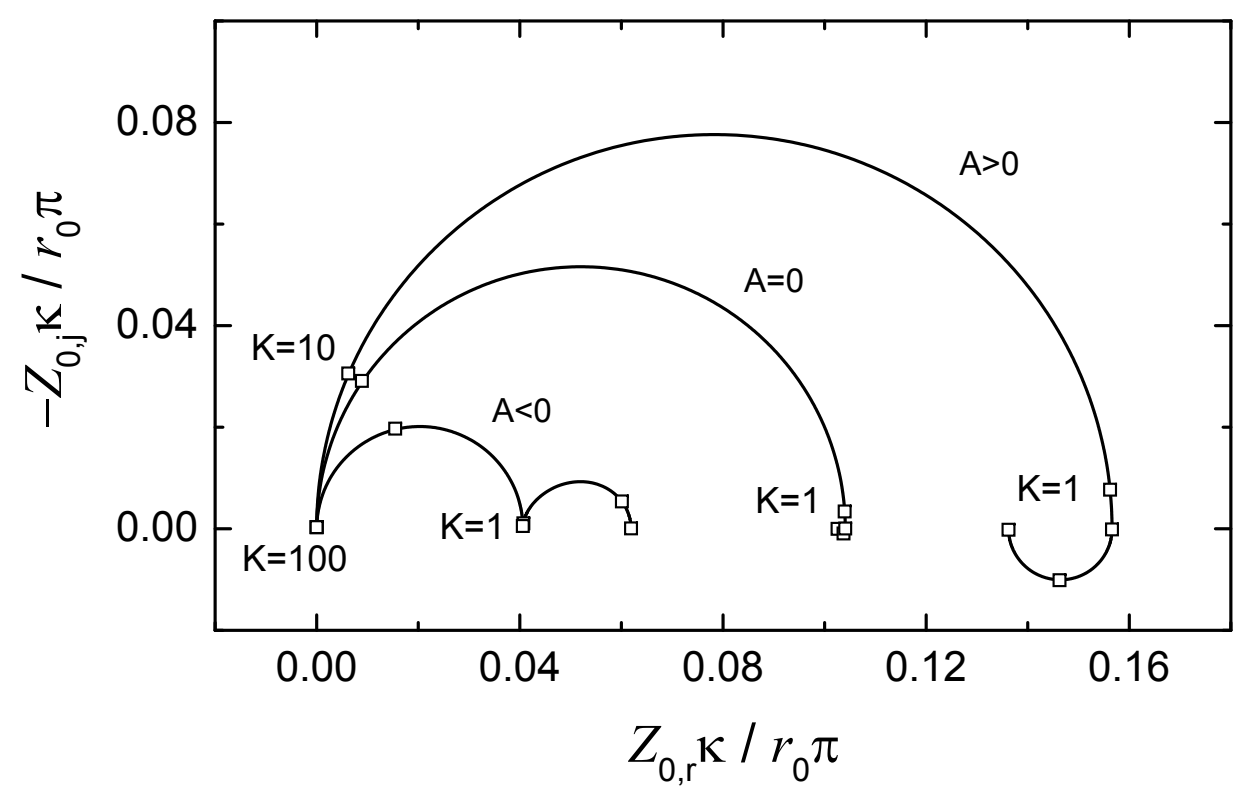

Figure 8: The global interfacial impedance for $\langle A\rangle>0,\langle A\rangle=0$, and $\langle A\rangle<0$ for the results presented in Figure 7 .

The faradaic impedance was calculated from equation (29) in terms of the surface-average parameters given from equation (30) to (32) and therefore did not account for the influence of electrode geometry. The geometry of the disk electrode is shown to distort the global impedance response. The geometry-induced distortion of impedance response and corresponding depressions of semicircles at high and low frequencies are more obvious in Figure $7(\mathrm{c})$ where $\langle A\rangle<0$.

The interfacial impedance corresponding to the calculations of Figure 7 are presented in Figure 8. No frequency dispersion is evident. The ohmic impedance, obtained by subtraction, is given in Figure 9. In contrast to the behavior seen in Figure 5, the effects of frequency dispersion is seen at both high and low frequencies. The overlapping of circles on the real axis represents the frequency range where the ohmic contribution may be expressed as a real number.

\subsection{Influence of Multi-Component Transport}

The normalized global impedances calculated under assumption of APS and NAPS are presented in Figures 10(a) and 10(b) for electrolytes containing $0.1 \mathrm{M} \mathrm{AgNO}_{3}$ and $1 \mathrm{M} \mathrm{KNO}_{3}$ and $0.01 \mathrm{M}$ $\mathrm{AgNO}_{3}$ and $1 \mathrm{M} \mathrm{KNO}_{3}$, respectively, where $K$ is given by equation (6). The difference between a priori and no-a-priori separation of faradaic and double-layer charging currents is observed at high frequencies where the faradaic and charging processes are both important. At high-frequencies, a depressed semicircle is seen for both the APS and the NAPS cases. The degree of depression, however, is greater for the NAPS case.

The distinction between the APS and NAPS models can be seen more clearly in Figure 11, where the logarithm of the absolute value of the imaginary part of the global impedance is presented as a function of the logarithm of frequency. As discussed by Orazem et al. [28], the slope of the curves presented in Figure 11, presented in Figure 12 with fraction of the mass-transfer-limited current 


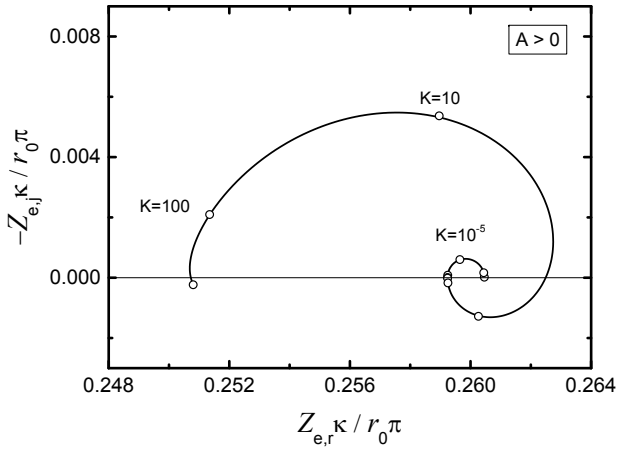

(a)

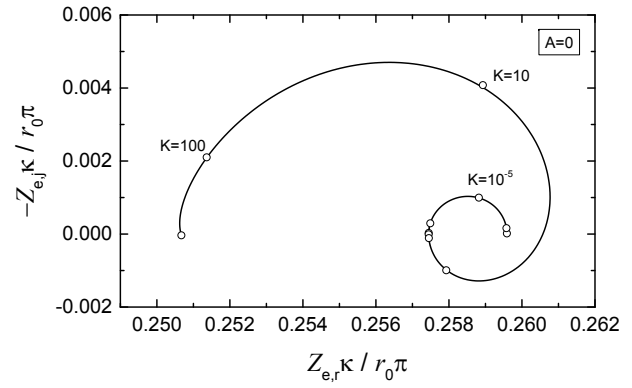

(b)

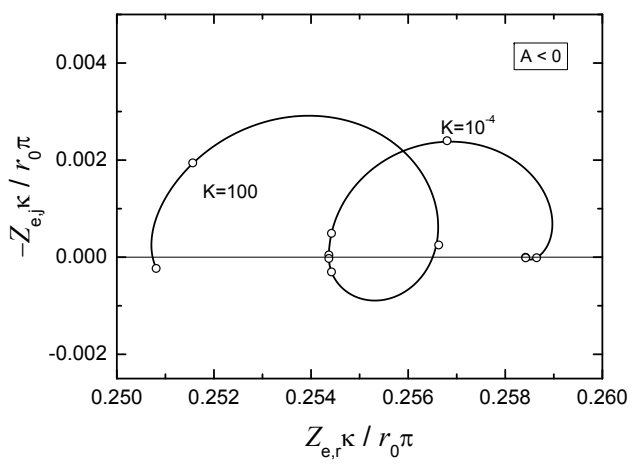

(c)

Figure 9: Nyquist representation of the calculated global ohmic impedance response for the results presented in Figure 7: a) $\langle A\rangle>0 ; \mathrm{b})\langle A\rangle=0$; and $(\mathrm{c})\langle A\rangle<0$.

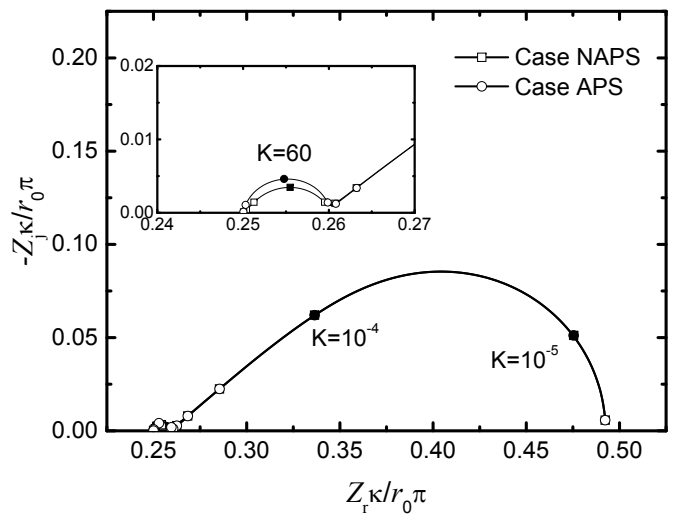

(a)

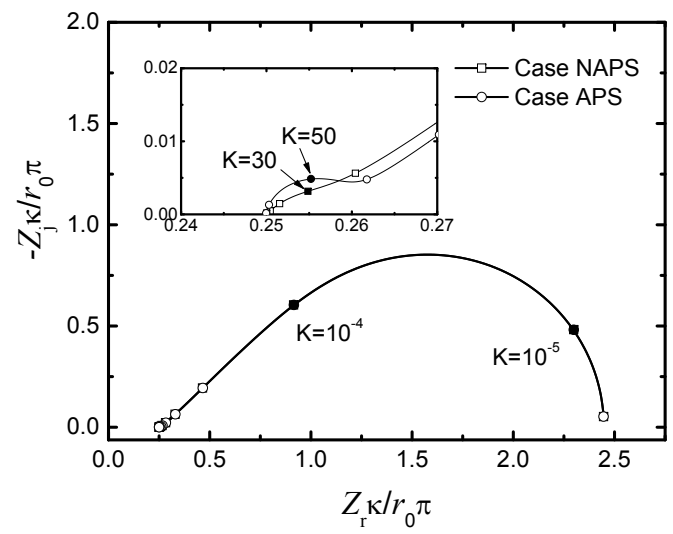

(b)

Figure 10: Calculated impedance response in Nyquist format for the case with a-priori separation (APS) and no a-priori separation (NAPS): a) $0.1 \mathrm{M} \mathrm{AgNO}_{3}$ in a $1 \mathrm{M} \mathrm{KNO}_{3}$ electrolyte; and b) $0.01 \mathrm{M} \mathrm{AgNO}_{3}$ in a $1 \mathrm{M} \mathrm{KNO}_{3}$ electrolyte. Taken from $\mathrm{Wu}$ et al. [12] 


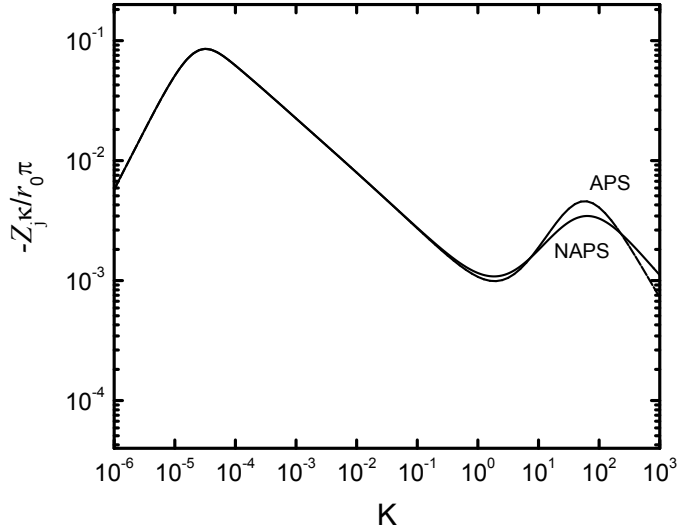

(a)

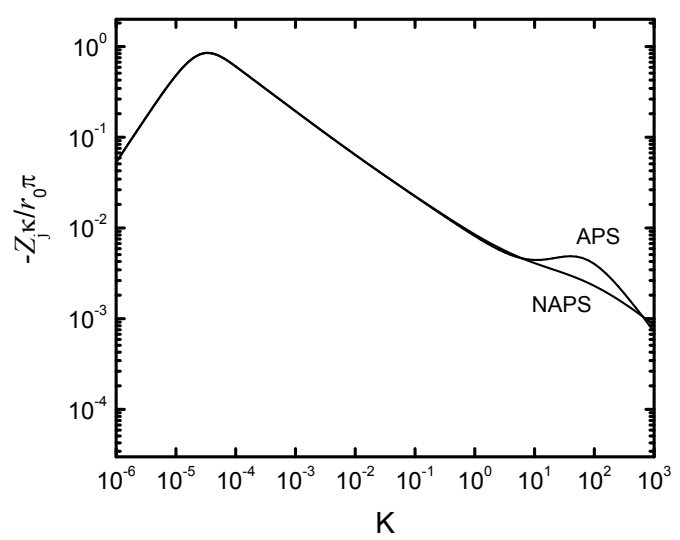

(b)

Figure 11: Calculated imaginary impedance response as a function of dimensionless frequency for the case with a-priori separation (APS) and no a-priori separation (NAPS): a) $0.1 \mathrm{M} \mathrm{AgNO}_{3}$ in a $1 \mathrm{M} \mathrm{KNO}_{3}$ electrolyte; and b) $0.01 \mathrm{M} \mathrm{AgNO}_{3}$ in a $1 \mathrm{M} \mathrm{KNO}_{3}$ electrolyte. Taken from Wu et al. [12]

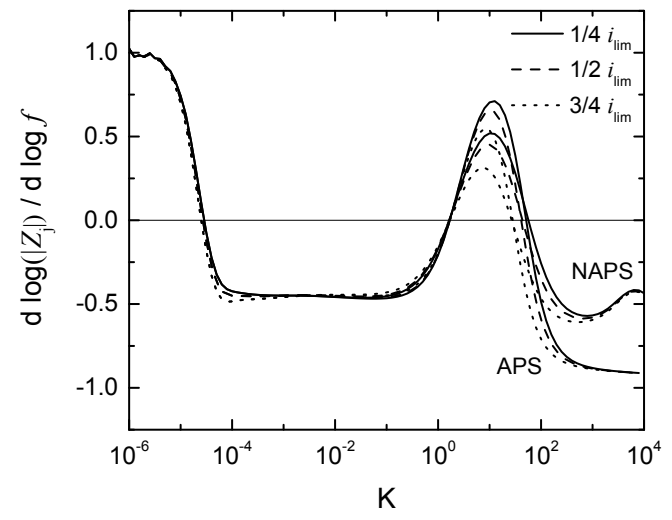

(a)

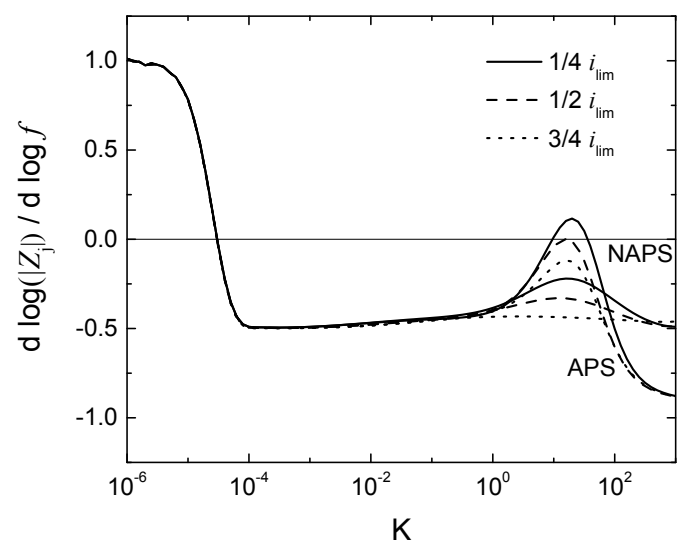

(b)

Figure 12: Calculated derivative of the logarithm of the imaginary part of the global impedance with respect to the logarithm of frequency for the case with a-priori separation (APS) and no a-priori separation (NAPS): a) 0.1 M $\mathrm{AgNO}_{3}$ in a $1 \mathrm{M} \mathrm{KNO}_{3}$ electrolyte; and b) $0.01 \mathrm{M} \mathrm{AgNO}_{3}$ in a $1 \mathrm{M} \mathrm{KNO}_{3}$ electrolyte. Taken from Wu et al. [12] 


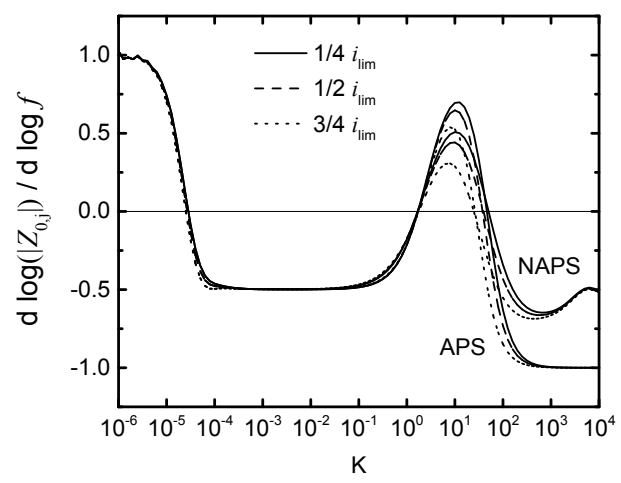

(a)

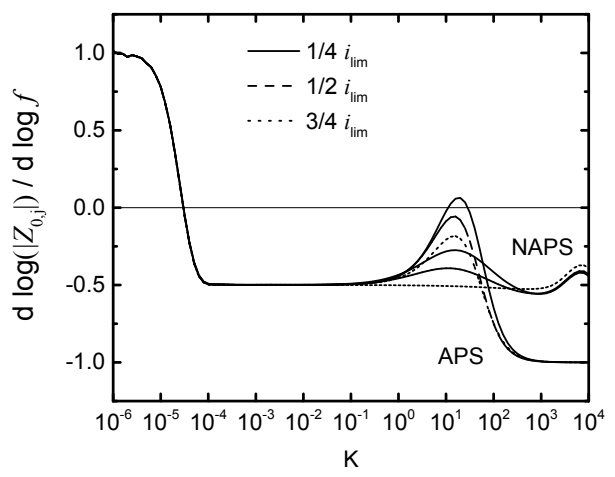

(b)

Figure 13: Calculated derivative of the logarithm of the imaginary part of the global interfacial impedance with respect to the logarithm of frequency for the case with a-priori separation (APS) and no a-priori separation (NAPS): a) $0.1 \mathrm{M} \mathrm{AgNO}_{3}$ in a $1 \mathrm{M} \mathrm{KNO}_{3}$ electrolyte; and b) $0.01 \mathrm{M} \mathrm{AgNO}_{3}$ in a $1 \mathrm{M} \mathrm{KNO}_{3}$ electrolyte.

density as a parameter, can be related to the exponent of a constant phase element.

The depressed semicircle obtained for the APS case is very similar to that obtained by Huang et al. [9] for a secondary current distribution in which mass transfer effects were ignored. The slope at high frequency has a mild frequency dependence and approaches a value of $\alpha=0.9$ for the APS results presented in both Figures 12(a) and 12(b). Huang et al. [9] reported similar values that were described as a pseudo-CPE behavior attributed to the influence of nonuniform current and potential distributions. The depressed semicircle obtained for the NAPS case corresponds to a highfrequency pseudo-CPE behavior with an exponent $\alpha$ between 0.5 and 0.6, which is much smaller than the values attributed to the influence of nonuniform current and potential distributions. [9]

The enhanced frequency dispersion associated with coupled charging and faradaic currents is seen by examination of the global interfacial impedance for the APS and NAPS cases, shown in Figure 13. Under the assumption that charging and faradaic current may be treated independently (APS), the derivative of the logarithm of the imaginary part of the global interfacial impedance with respect to the logarithm of frequency approaches -1 at high frequencies, as would be expected for an ideal faradaic reaction. In sharp contrast, the corresponding value obtained for the NAPS case is between -0.7 and -0.5 . These figures suggest that an additional factor must be contributing to the frequency dispersion when charging and faradaic currents are assumed to be coupled (e.g., NAPS).

The simulations of the coupled charging and faradaic currents requires thermodynamic properties of the interface that are not readily available. For the present work, these were obtained under assumption of a Stern-Gouy-Chapman model of a diffuse double layer in the absence of ion-specific adsorption. Use of a refined model will certainly alter the results. The work serves to show, however, that the frequency dispersion associated with the coupling of charging and faradaic currents does not disappear in the presence of a large concentration of supporting electrolyte. This result contradicts the conclusions presented by Nisancioglu and Newman.[29] Even though a low concentration of silver ions must be associated with a small contribution to the charging current, the contribution to the charging current influences the contribution to the faradaic current and, therefore, influences the impedance response. 
The controversy over whether one may decouple faradaic and charging currents that was expressed in the 1960s by papers by Sluyters[30] and Delahay[31] was recently resurrected by the work of Nisancioglu and Newman.[29] The coupling of charging and faradaic currents arises from the contribution of the flux of reacting species to charging the interface as well as to the faradaic reaction. It is evidently appropriate to decouple faradaic and charging currents for systems that are not influenced by mass transfer. The present work suggests, however, that the coupling of faradaic and charging currents should be considered when modeling the impedance response for systems influenced by mass transfer, even for large concentrations of supporting electrolyte. The present work shows that the coupling of faradaic and charging currents results in a frequency dispersion that may appear as a pseudo-CPE behavior.

\section{Conclusions}

Numerical simulations of the impedance response for a disk geometry must be performed with care. The results of these simulations provides valuable insight that facilitates interpretation of experimental impedance spectra. in addition, the appreciation of the characteristic frequencies at which frequency dispersion appears may be used to facilitate electrode design. The work, however, also suggests that more effort is needed to understand the role of mass transfer on impedance response.

Jorcin et al. [32] suggested that CPE behavior, seen in global measurements, can be attributed to time-constants that are distributed either along the electrode surface or in the direction perpendicular (or normal) to the electrode surface. In some cases, the distributions of time constants results in pseudo-CPE behavior in which CPE parameters may appear as functions of frequency. Such was the case for the impedance response reported by Huang et al. [7-9] that was affected nonuniform current and potential distributions. Huang et al. [7] showed that their distributions could be attributed to a surface distribution of time constants. The frequency dispersion associated with coupling of faradaic and charging currents cannot be characterized as being associated with either surface or normal distributions. More work is needed to facilitate interpretation of this source of frequency dispersion.

\section{Acknowledgements}

The work described here took place over a decade. The authors gratefully acknowledge the financial support of their home institutions, which facilitated this collaboration. 


\section{References}

[1] J. S. Newman, Resistance for flow of current to a disk, J. Electrochem. Soc. 113 (5) (1966) $501-502$.

[2] J. S. Newman, Current distribution on a rotating disk below the limiting current, J. Electrochem. Soc. 113 (1966) 1235-1241.

[3] K. Nisancioglu, J. S. Newman, The transient response of a disk electrode, J. Electrochem. Soc. 120 (1973) 1339-1346.

[4] K. Nisancioglu, J. S. Newman, The short-time response of a disk electrode, J. Electrochem. Soc. 121 (1974) 523-527.

[5] P. Antohi, D. A. Scherson, Current distribution at a disk electrode during a current pulse, J. Electrochem. Soc. 153 (2006) E17-E24.

[6] J. S. Newman, Frequency dispersion in capacity measurements at a disk electrode, J. Electrochem. Soc. 117 (1970) 198-203.

[7] V. M.-W. Huang, V. Vivier, M. E. Orazem, N. Pébère, B. Tribollet, The apparent CPE behavior of an ideally polarized disk electrode: A global and local impedance analysis, J. Electrochem. Soc. 154 (2007) C81-C88.

[8] V. M.-W. Huang, V. Vivier, I. Frateur, M. E. Orazem, B. Tribollet, The global and local impedance response of a blocking disk electrode with local CPE behavior, J. Electrochem. Soc. 154 (2007) C89-C98.

[9] V. M.-W. Huang, V. Vivier, M. E. Orazem, N. Pébère, B. Tribollet, The apparent CPE behavior of a disk electrode with faradaic reactions, J. Electrochem. Soc. 154 (2007) C99 C107.

[10] S.-L. Wu, M. E. Orazem, B. Tribollet, V. Vivier, Impedance of a disk electrode with reactions involving an adsorbed intermediate: Local and global analysis, J. Electrochem. Soc. 156 (1) (2009) C28-C38.

[11] S.-L. Wu, M. E. Orazem, B. Tribollet, V. Vivier, Impedance of a disk electrode with reactions involving an adsorbed intermediate: Experimental and simulation analysis, J. Electrochem. Soc. 156 (7) (2009) C214-C221.

[12] S.-L. Wu, M. E. Orazem, B. Tribollet, V. Vivier, The influence of coupled faradaic and charging currents on impedance spectroscopy, Electrochimica Acta 131 (2014) 3-12.

[13] C. Blanc, M. Orazem, N. Pbre, B. Tribollet, V. Vivier, S. Wu, The origin of the complex character of the ohmic impedance, Electrochim. Acta 55 (2010) 6313-6321.

[14] J. V. Ferrari, H. G. D. Melo, M. Keddam, M. E. Orazem, N. Pébère, B. Tribollet, V. Vivier, Influence of normal and radial contributions of local current density on local electrochemical impedance spectroscopy, Electrochim. Acta 60 (2012) 244-252. 
[15] I. Frateur, V. M.-W. Huang, M. E. Orazem, N. Pébère, B. Tribollet, V. Vivier, Local electrochemical impedance spectroscopy: Considerations about the cell geometry, Electrochim. Acta 53 (2008) 7386-7395.

[16] V. M. Huang, S.-L. Wu, M. E. Orazem, N. Pébère, B. Tribollet, V. Vivier, Local electrochemical impedance spectroscopy: A review and some recent developments, Electrochim. Acta 56 (23) (2011) $8048-8057$.

[17] C. Wagner, Theoretical analysis of the current density distribution in electrolytic cells, J. Electrochem. Soc. 98 (1951) 116-128.

[18] I. Epelboin, M. Keddam, Faradaic impedances: Diffusion impedance and reaction impedance, Journal of the Electrochemical Society 117 (1970) 1052-1056.

[19] L. Péter, J. Arai, H. Akahoshi, Impedance of a reaction involving two adsorbed intermediates: Aluminum dissolution in non-aqueous lithium imide solutions, J. Electroanal. Chem. 482 (2) (2000) $125-138$.

[20] R. D. Armstrong, K. Edmondson, The impedance of metals in the passive and transpassive regions, Electrochim. Acta 18 (1973) 937-943.

[21] C. Cao, On the impedance plane displays for irreversible electrode reactions based on the stability conditions of the steady-statei. one state variable besides electrode potential, Electrochim. Acta 35 (1990) 831-836.

[22] I. Epelboin, M. Keddam, J. C. Lestrade, Faradaic impedances and intermediates in electrochemical reactions, Faraday Discuss. 56 (1973) 264-275.

[23] J. S. Newman, K. E. Thomas-Alyea, Electrochemical Systems, 3rd Edition, John Wiley \& Sons, Hoboken, NJ, 2004.

[24] M. E. Orazem, B. Tribollet, Electrochemical Impedance Spectroscopy, John Wiley \& Sons, Hoboken, NJ, 2008.

[25] G. Sewell, The Numerical Solution of Ordinary and Partial Differential Equations, John Wiley \& Sons, New York, 2005.

[26] J. O. Bockris, A. K. N. Reddy, Modern electrochemistry: An introduction to an interdisciplinary area, Plenum Press, New York, 1970.

[27] W. G. Cochran, The flow due to a rotating disc, Proceedings of the Cambridge Philosophical Society 30 (1934) 365-375.

[28] M. E. Orazem, N. Pébère, B. Tribollet, Enhanced graphical representation of electrochemical impedance data, J. Electrochem. Soc. 153 (2006) B129-B136.

[29] K. Nisancioglu, J. S. Newman, Separation of double-layer charging and faradaic processes at electrodes, J. Electrochem. Soc. 159 (2012) E59-E61.

[30] J. H. Sluyters, On the impedance of galvanic cells I. theory, Recl. Trav. Chim. Pays-Bas 79 (1960) 1092-1100. 
[31] P. Delahay, Electrode processes without a priori separation of double-layer charging, J. Phys. Chem. 70 (7) (1966) 2373-2379.

[32] J.-B. Jorcin, M. E. Orazem, N. Pébère, B. Tribollet, CPE analysis by local electrochemical impedance spectroscopy, Electrochim. Acta 51 (2006) 1473-1479. 